\title{
Alexitimia y síndrome de Asperger
}

\author{
Isabel Paula-Pérez, Juan Martos-Pérez, María Llorente-Comí
}

Introducción. El estudio parte de la hipótesis que sostiene que la dificultad para identificar y describir emociones y sentimientos, y para diferenciar los sentimientos de las sensaciones corporales que los acompañan, se configuran en denominador común del constructo de alexitimia y del síndrome de Asperger (SA).

Pacientes y métodos. La investigación evalúa los niveles de alexitimia en nueve varones adultos con SA.

Resultados. Los resultados se clasifican en función de si la fuente de información es el propio sujeto con SA (nivel de alexitimia significativo en dos tercios de los sujetos encuestados) o si dicha fuente es un familiar (puntuación final en relación a la presencia de alexitima el doble que en la población neurotípica).

Conclusión. En base a los adultos estudiados se confirma una comorbilidad significativa entre alexitimia y SA que conduce al cuestionamiento de si la alexitimia es un rasgo idiosincrásico de los sujetos con SA.

Palabras clave. Adultos con síndrome de Asperger. Alexitimia.

\section{Introducción}

\section{El constructo 'alexitimia'}

Aunque en los años cuarenta y cincuenta algunos autores intentaban dar cuenta del carácter vago e impersonal del discurso de los pacientes psicosomáticos al tratar como situaciones normales situaciones de intenso estrés emocional, no es hasta 1973 cuando el psiquiatra Peter E. Sifneos [1], en el ámbito de la clínica de pacientes con estos trastornos en el Beth Israel Hospital, acuña el término 'alexitimia' -de las raíces griegas $a$ (sin), lexis (palabra) y thimos (afecto), es decir, 'sin palabras para el afecto'- para dar cuenta de las serias dificultades que presentaban en hablar sobre sus emociones, identificar el componente afectivo de éstas, la incapacidad para expresarlas verbalmente, las dificultades en el uso de la fantasía y la postura rígida que adoptaban.

Sifneos [2] postuló dos tipos de alexitimia: primaria y secundaria. La alexitimia primaria, de orden biológico, es un defecto estructural neuroanatómico o una deficiencia neurobiológica en la forma de anormalidades, debidas a factores hereditarios que interrumpen la comunicación entre el sistema límbico y la neocorteza. En este caso, la alexitimia es una disposición personal, que acompaña al sujeto de por vida, como rasgo de personalidad fruto de una anormalidad genética, un desarrollo biológico inadecuado o una lesión cerebral. Además, Sifneos añade que la especialización de los hemisferios ce- rebrales también desempeña un papel importante cuando se produce una inadecuada comunicación entre el hemisferio izquierdo, que se encarga de la elaboración y expresión del lenguaje articulado, y el hemisferio derecho, que modula las emociones y proporciona al lenguaje los aspectos prosódicos de entonación, coloratura, cadencia y melodía. La alexitimia secundaria se origina como resultado de influencias psicológicas, como condicionamiento sociocultural o defensa ante un trauma. La persona suprime las emociones dolorosas como un mecanismo de defensa contra el trauma, por ejemplo, enfermedades crónicas graves, duelo por un ser querido, etc. Posiblemente se relacione con una negación de la situación o acontecimiento traumático para protegerse de emociones demasiado dolorosas. En estos casos, la alexitimia puede desaparecer cuando desaparece la enfermedad, se elabora el duelo o la situación traumática, o puede cronificarse si no se interviene adecuadamente.

Como esta distinción resulta a veces ambigua, muchos autores prefieren no utilizarla. Esto se debe a que las causas psicológicas y neurológicas pueden estar interconectadas, influyéndose mutuamente. No obstante, puede ser importante establecer una distinción entre una alexitimia en la que las estructuras neurológicas vinculadas con las emociones están dañadas o destruidas, y aquella en la que sólo se da una disfunción (p. ej., una inhibición de la actividad neuronal).

La alexitima no aparece como constructo en las clasificaciones psiquiátricas, como el Manual diag-
Departamento de Métodos de Investigación y Diagnóstico en Educación; Universitat de Barcelona (I. Paula-Pérez) Centro DELETREA (Diagnóstico, Evaluación del Lenguaje y Tratamiento del Espectro Autista); Madrid, España (J. Martos-Pérez, M. Llorente-Comí)

Correspondencia:

Dra. Isabel Paula Pérez. Campus Mundet. Edificio Llevant, 2. piso, despacho 264. Pg. Vall d'Hebron, 171. E-08035 Barcelona.

E-mail:

isabelpaula@ub.edu

Agradecimientos:

A J.J. Ordinas y R. Nogués, por la traducción de varios de los instrumentos utilizados y la facilitación, siempre generosa, de la información a través de su página web (http://espectroautista.info).

Aceptado:

24.12.09.

Cómo citar este artículo: Paula-Pérez I, Martos-Pérez J, Llorente-Comí M. Alexitimia y síndrome de Asperger. Rev Neurol 2010; 50 (Supl 3): S85-90.

(C) 2010 Revista de Neurología 
Tabla I. Características de la alexitimia.

Dificultad para identificar emociones y sentimientos

Dificultad para diferenciar una emoción de otra. Esta incapacidad no se da sólo respecto a los propios sentimientos, sino también respecto a las emociones en los demás (en sus voces, expresiones faciales, posturas)

Dificultad para describir emociones y sentimientos

Dificultad para describir lo que sienten y describir a otras personas todo lo referente al ámbito subjetivo y de los afectos

Dificultad para diferenciar los sentimientos de las sensaciones corporales que acompañan a la activación emocional

Las emociones suelen acompañarse de síntomas fisiológicos, como sudoración, aceleración del ritmo cardiaco, etc. Las personas con alexitimia atribuyen estas manifestaciones fisiológicas a síntomas vagos o los confunden con la emoción misma. Cuando sienten emociones intensas, estas personas describe simplemente un malestar físico de un modo impreciso

Reducida capacidad de fantasía y de pensamiento simbólico

Tendencia al pensamiento concreto, con problemas para el manejo simbólico de las emociones. Forma de hablar monótona, parca y sin matices afectivos. Apenas gesticulan ni introducen cambios en el tono de voz, se sientan de forma rígida y el semblante es inexpresivo

Utilizan la acción como estrategia de afrontamiento en situaciones de conflicto

El modo de resolver un estado emocional desagradable consiste en la realización de conductas directas. Tienen una orientación de tipo práctico y un escaso contacto con su realidad psíquica

nóstico y estadístico de los trastornos mentales (DSMIV), pero ha demostrado tener un valor heurístico importante, aceptado por la comunidad científica y susceptible de investigarse. La alexitimia se trata más bien de un tipo de sintomatología que puede aparecer en personas con una amplia variedad de diagnósticos clínicos. Además, no todas las personas tienen el mismo grado de afectación, por lo que es importante no considerarla como un síntoma de 'todo o nada'.

Dado que en muchas personas la alexitimia aparece de una manera muy leve, se ha abierto un debate en torno a la alexitimia como rasgo de personalidad o la alexitimia como estado (Tabla I).

Thompson [3] describe las características de la alexitimia en base a los estudios de Taylor et al [4]:

\section{Dificultad para identificar y describir sentimientos} Las personas con alexitimia manifiestan una dificultad para reconocer estados emocionales que están sucediendo. La mayoría de ellos pueden tener una sensación de incomodidad, de que algo está cambiando somáticamente (rubor, cosquilleo en el estómago, aumento de la tasa cardíaca, etc.), pero cuando se les pide que expliquen sobre sus sentimientos, la persona no tiene palabras que ofrecer, como mucho una respuesta forzada o simplemente lo que hace es cambiar de tema. A menudo, el sujeto malinterpreta la expresión física de lo emocional como una expresión física de enfermedad: por ejemplo, las lágrimas en la mejilla se convierten en un defecto del lagrimal y no en tristeza; la pasión, en una válvula del corazón defectuosa; la ansiedad en el estómago, en una apendicitis... Además, los estados emocionales pueden atribuirse a influencias ambientales adversas (cambios en la presión atmosférica, tóxicos en el aire, un colchón incómodo, etc.). Es como si hubiera una falta de relación que permita a la imaginación formarse un cuadro de la situación emocional. De ahí la importancia de la imaginación y, como dice Aristóteles, 'la mente nunca piensa sin una imagen.'

\section{Procesos imaginativos restringidos}

Entendemos la imaginación como la capacidad para formarnos imágenes en nuestra mente a partir del recuerdo de aspectos concretos de las experiencias sensoriales. Sin embargo, una evaluación más profunda muestra que la imaginación también es capaz de crear nuevas imágenes nunca antes vistas en el mundo sensorial concreto. Así pues, la imaginación sirve para más funciones, como la habilidad para imaginar qué son nuestras emociones, deseos, necesidades y cómo nos gustaría cumplirlos, cómo podemos imaginar los conflictos presentes y pasados, cómo podemos regular el tipo y la intensidad de nuestra expresión emocional, y cómo imaginarnos a nosotros mismos en la piel de otra persona. Esa es la base de la empatía y de la capacidad para ser efectivo en la modulación de los estados emocionales de los demás.

\section{Estilo cognitivo orientado a aspectos externos}

Debido a las dificultades para reconocer su mundo interior de sentimientos, las personas con alexitimia tienden a orientar su existencia hacia otras áreas en las que se sienten más competentes, como el mundo físico concreto. Incluso pueden llegar a ser extraordinarios en el 'mundo de las cosas', de los objetos materiales, de los hechos empíricos.

\section{Alexitimia y síndrome de Asperger}

Cada vez cobra mayor fuerza la hipótesis de que la alexitimia es uno de los constructos que mide de una manera precisa y científica las diferencias emocionales inherentes al síndrome de Asperger (SA). Llama poderosamente la atención la yuxtaposición existente entre los síntomas prototípicos de la alexitimia y los del SA en cuanto a:

- Alteraciones cognitivas. Problemas de introspección, pobre capacidad imaginativa, pensamiento 
concreto y operatorio, y sensación de afecto plano y aburrido aun cuando pueden ser personas inteligentes e intelectuales.

- Alteraciones de las relaciones interpersonales. Dificultades para identificar adecuadamente sus propios sentimientos, pobre comunicación verbal de su estrés emocional a otras personas, dificultad para ver a los demás como fuente de ayuda, preferencia por estar solos y evitar a la gente o carencia de interés por las personas que le rodean, y una reducción importante de intereses compartidos. Pueden mostrar temor en situaciones de intimidad, tratar a los demás como si fueran objetos, falta de empatía, dificultades para descifrar el comportamiento no verbal y problemas para comprender grupos sociales.

- Alteraciones del discurso y el lenguaje. Dificultades en el uso de metáforas, lenguaje plano, banal y basado en hechos. Casi ausencia completa de pensamientos basados en actitudes interiores, sentimientos, deseos. Relato detallado y a menudo aburrido de acontecimientos y de su actuación en ellos. Suele haber un uso reducido del pronombre 'yo' y muestran una clara dificultad en la función simbólica. El contenido de la comunicación y la prosodia es pobre, y el tono de voz, monótono. Pueden aprender respuestas emocionales apropiadas, pero a menudo son demasiado formales y pedantes.

- Alteraciones del comportamiento no verbal. Dificultades para identificar las emociones a través de los gestos faciales y de la comunicación no verbal.

\section{Alexitimia y toma de decisiones}

Las emociones son muy importantes a la hora de tomar decisiones con efectividad. Esto se debe a dos factores principales:

- Las emociones desempeñan un papel fundamental para determinar nuestras metas en base a lo que nos agrada o desagrada. Si no somos capaces de reconocer nuestras emociones e interpretarlas, no estaremos utilizando una información muy valiosa. Las personas con alexitimia basan sus decisiones en razones prácticas, no sentimentales ni hedonistas, de modo que llevan vidas menos gratificantes y placenteras.

- El sistema emocional utiliza un modo diferente de procesar la información que el sistema intelectual. Es más holista y distribuido, se especializa en reacciones rápidas y funciona de un modo automático, basado en el conocimiento adquirido en experiencias previas. En cambio, el intelecto progresa paso a paso, según una secuencia lógi- ca, pero requiere mucho más tiempo para procesar la información y puede dejar de lado información importante. Ambos métodos tienen sus ventajas y sus inconvenientes y la mayoría de las personas utiliza ambos en la toma de decisiones o utiliza el que considera más adecuado en cada momento. Las personas con alexitimia emplean sólo el sistema intelectual, no son intuitivos y se ven fácilmente abrumados por tareas prácticas o razonamientos interminables. Por ejemplo, si quieres comprar un automóvil y no eres capaz de decidirte al analizar las prestaciones de diversos modelos, es posible que optes por el que te produzca una sensación más agradable, mientras que la persona con alexitimia no hará esto y seguirá teniendo problemas para tomar una decisión. Los sujetos con alexitimia suelen ser muy indecisos cuando han de tomar decisiones respecto a preferencias personales. Al no ser capaces de decidir en función de la emoción que algo les inspira, pueden tender a buscar la opinión de los demás y decidir en base a ello.

\section{Pacientes y métodos}

\section{Muestra}

Han participado nueve sujetos, todos ellos varones adultos con diagnóstico de SA realizado por profesionales con dilatada experiencia de acuerdo con los criterios diagnósticos del DSM-IV-TR [5]. El rango de edad, en el momento del estudio, se sitúa entre 20 y 43 años (media de edad: 28,33 \pm 8,21 años). La mayor parte de ellos con estudios universitarios. Con empleo en un solo caso. La mayor parte recibían tratamiento psicológico y farmacológico (Tabla II).

\section{Instrumentos y procedimiento}

Se han utilizado las siguientes escalas y cuestionarios (con la muestra también se han utilizado otros instrumentos que no se recogen en el estudio actual):

\section{Escala de alexitimia de Toronto (TAS-20)}

La TAS fue desarrollada por Bagby et al [6] y procede de dos versiones anteriores de los mismos autores con mayor número de ítems. En España, existen dos validaciones: la de Moral de la Rubia y Retamales [7] y la de Martínez-Sánchez [8]. El más actual, el primero, se trata de un cuestionario autoadministrado que consta de 20 preguntas con seis posibilidades de respuesta, desde 'muy en desacuerdo' hasta 'muy de acuerdo', y que se puntúan entre 0 y 5 . 
Tabla II. Edad, profesión/estudios y tratamiento de los sujetos.

\begin{tabular}{|c|c|c|c|c|}
\hline Sujeto & Edad (años) & Profesión/estudios & Medicación & Tratamiento psicológico \\
\hline 1 & 28 & Delineante/COU & Risperdal, Seroxat & Deletrea \\
\hline 2 & 21 & Grado superior FP & & Deletrea \\
\hline 3 & 28 & Doctorado en Física & Tryptizol (ocasional) & Deletrea \\
\hline 4 & 25 & Estudiante de Biología & $\begin{array}{l}\text { Citalopram, Abilify, } \\
\text { Lornetocepan }\end{array}$ & Deletrea \\
\hline 5 & 31 & Licenciado en Historia & Fluoxetina & Asociación Asperger Aragón \\
\hline 6 & 39 & Informático & Paroxitina & Psicoterapia privada \\
\hline 7 & 43 & $\begin{array}{l}\text { Estudiante de Formación } \\
\text { del Profesorado }\end{array}$ & Cymbalta, Risperdal & Psicoterapia privada \\
\hline 8 & 20 & $\begin{array}{l}\text { Técnico de Gestión } \\
\text { Comercial }\end{array}$ & $\begin{array}{l}\text { Prozac, Estratera, } \\
\text { Eutirox }\end{array}$ & Deletrea \\
\hline 9 & 20 & Bachillerato & & Deletrea, Seguridad Social \\
\hline
\end{tabular}

Las cuestiones 4, 5, 10, 18 y 19 puntúan de forma inversa. Existe una estructura ortogonal tridimensional subyacente que explica el $31 \%$ de la varianza.

Las tres subescalas son: dificultad para la discriminación de señales emocionales (ítems 1, 3, 6, 7, 9,13 y 14), dificultad en la expresión verbal de las emociones (ítems $2,4,11,12$ y 17) y pensamiento orientado hacia detalles externos (ítems 5, 8, 10, 15, $16,18,19$ y 20$)$.

El rango de resultados se extiende de 20 a 100. Aunque los autores no indican puntos de corte, en la validación española se demostró la validez discriminante del instrumento al ser altamente significativa la diferencia de puntuación observada entre la muestra clínica $(51,82)$ y la muestra control $(44,23)$. Se proponen las siguientes puntuaciones para interpretar los resultados: $\leq 51$, ausencia de alexitimia; entre 51 y 60, posible alexitimia, $y \geq 61$, alexitimia.

\section{Cuestionario de alexitimia en línea (OAQ-G2)}

El OAQ-G2 fue desarrollado en el año 2005 por Thompson [9] como respuesta a las limitaciones observadas en los otros cuestionarios de alexitimia existentes. La puntuación máxima posible es de 185 y el autor sugiere las siguientes puntuaciones para interpretar los resultados: $\leq 94$, ausencia de alexitimia; entre 95 y 112, posible alexitimia, $y \geq 113$, alexitimia.

Forzar a proporcionar una respuesta a todas las preguntas puede ser frustrante porque puede darse el caso de que una pregunta no se pueda aplicar a la persona que realiza la prueba. Por esta razón, a las preguntas sin responder se les asigna automáticamente la respuesta 'indeciso'. Hay que responder las preguntas de la forma más espontánea posible y si no se sabe qué contestar en alguna pregunta, hay que ignorarla y continuar con la siguiente. Cada pregunta puede tener una respuesta a escoger de entre cinco posibilidades.

\section{Escala de alexitimia para observadores (OAS)}

La OAS se ha utilizado en distintas publicaciones, por ejemplo, por Haviland et al [10]. El cuestionario se ha traducido directamente de su fuente original y está pensado para ser contestada por familiares o conocidos de la persona a quien se desea evaluar. La escala se divide en cinco subescalas:

- Subescala 1: distante (10 preguntas: 1 a 10).

- Subescala 2: no introspectivo (8 preguntas: 11 a 18).

- Subescala 3: somatización (5 preguntas: 19 a 23).

- Subescala 4: sin sentido del humor (5 preguntas: 24 a 28).

- Subescala 5: rígido (5 preguntas: 29 a 33).

La OAS consta de 33 preguntas, puntuables entre 0 y 3, lo que permite una puntuación máxima de 99 . Los autores de la OAS no han establecido puntos de corte, es decir, puntuaciones que permitan clasificar el nivel de alexitimia según la puntuación obtenida. De lo que disponemos es del resultado obtenido al enfrentar al cuestionario a población normal y a población clínica. La población normal utilizada en el estudio de validación de la OAS estaba formada por familiares de universitarios de Ciencias de la Salud, mientras que la población clínica consistía en pacientes de psicólogos afiliados a la Asociación Psicológica Americana. Es de prever que en la población general se encuentren algunos casos de individuos alexitímicos, pero pocos, mientras que en la población clínica es de prever la situación inversa, es decir, que el número de personas con alexitimia sea significativo.

\section{Resultados}

Con la TAS-20, 5 sujetos (55\%) obtienen una puntuación que indica presencia de alexitimia, 1 sujeto (11\%) obtiene una puntuación que sugiere posible alexitimia, y en 3 casos (34\%) la puntuación indica ausencia de alexitimia. Es decir, con este instrumento, dos tercios de los sujetos presentan alexitimia.

Con el OAQ-G2, 3 sujetos (34\%) obtienen una puntuación que indica clara presencia de alexitimia, 4 sujetos (44\%) obtienen una puntuación que sugiere posible alexitimia, y en 2 sujetos (22\%) la 
puntuación indica ausencia de alexitimia. Con este instrumento y sumando los dos primeros grupos de personas, tres cuartes partes de los sujetos presentan o pueden presentar alexitimia.

Con la OAS, los datos de las medias (totales y en las distintas subescalas) de la población normal y clínica que proporcionan los autores de la escala son los datos obtenidos en las respuestas de los siete familiares de sujetos con SA que han respondido. Los resultados muestran que, en el SA, las medias son incluso mayores que las encontradas en la población clínica (con la excepción de la subescala de somatización, que es inferior, pero algo superior que la obtenida en la población normal, y de la subescala distante, que es ligeramente inferior a la obtenida en la población clínica). Las personas con SA obtienen medias muy superiores, prácticamente el doble (con la excepción de la subescala de somatización, que es ligeramente superior) a las obtenidas en la población normal (Tabla III).

\section{Discusión}

La investigación revela una superposición entre alexitimia y SA. Los datos obtenidos en la muestra concuerdan con los obtenidos por Hill et al [12], quienes hallaron que el $85 \%$ de las personas con SA mostraban una alexitimia moderada o grave.

El análisis global de los datos incita a una diferenciación de los resultados en función de si la fuente de información es el propio sujeto con SA (TAS-20 y OAQ-G2) o si dicha fuente es un familiar que convive con la persona con SA (OAS). En el primero de los casos, los datos son significativos pero no concluyentes: dos tercios de los sujetos con SA presentan o pueden presentar alexitimia, según la TAS-20, y tres cuartas partes presentan o pueden presentar alexitimia, según el OAQ-G2. Sin embargo, cuando se trata de valorar los niveles de alexitimia por parte de los familiares, los datos son determinantes, doblando la puntuación obtenida en la población neurotípica.

Una hipótesis al respecto podría ser la propia dificultad que halla el sujeto con SA para responder a los cuestionarios seleccionados, dificultad inherente a su capacidad introspectiva para describir y analizar los sentimientos y emociones propios y ajenos. Los cuestionarios exigen, justamente, autoinformar en relación a parámetros socioemocionales confusos para personas con SA: descripción de emociones, significado de las reacciones emocionales de los demás, fantasear sobre proyectos personales o deseos, etc.

La desregulación emocional en los casos estudiados incluye: dificultades para identificar y describir
Tabla III. Puntuaciones medias ( \pm desviación estándar) totales y en las distintas subescalas en población normal, población clínica y síndrome de Asperger en la OAS.

\begin{tabular}{lccc}
\hline & $\begin{array}{c}\text { Hombres } \\
\text { (población normal) }\end{array}$ & $\begin{array}{c}\text { Hombres } \\
\text { (población clínica) }\end{array}$ & $\begin{array}{c}\text { Síndrome } \\
\text { de Asperger }\end{array}$ \\
\hline Total & $28,5 \pm 12,8$ & $50,0 \pm 14,8$ & $54,4 \pm 16,5$ \\
\hline Subescala 1 (distante) & $9,5 \pm 5,5$ & $17,1 \pm 5,7$ & $16,2 \pm 5,8$ \\
\hline Subescala 2 (no introspectivo) & $8,3 \pm 4,4$ & $13,1 \pm 4,1$ & $17,7 \pm 4,6$ \\
\hline Subescala 3 (somatización) & $4,0 \pm 2,6$ & $6,8 \pm 4,6$ & $4,5 \pm 2,1$ \\
\hline Subescala 4 (sin sentido del humor) & $2,5 \pm 2,7$ & $6,9 \pm 3,4$ & $7,7 \pm 3,1$ \\
\hline Subescala 5 (rígido) & $4,1 \pm 3,2$ & $6,2 \pm 3,3$ & $9,5 \pm 2,5$ \\
\hline
\end{tabular}

emociones, dificultades para distinguir las emociones de las sensaciones corporales que las acompañan, alteraciones en los procesos de imaginación y fantasías relacionados con las competencias simbólicas, una tendencia a centrarse en los acontecimientos externos más que en las experiencias internas, como las emociones y los pensamientos, y el peso de la lógica antepuesta a los sentimientos en la toma de decisiones. Estos resultados pueden relacionarse con los recientes hallazgos de Lombardo et al [13], quienes confirman que el cerebro de las personas con trastornos del espectro autista es menos activo cuando éstas se ven involucradas en pensamientos autorreflexivos y tienen problemas cuando se trata de pensar o reflexionar sobre sí mismas.

Como se han detectado altas puntuaciones en la dificultad para encontrar las palabras correctas para sus sentimientos, para analizar los problemas, para saber lo que pasa dentro de sí mismas y para estar en contacto con las emociones, es posible que encuentren confuso y ambiguo contestar a los cuestionarios presentados y en nuestra muestra expresaron dificultades para comprender algunos de los ítems. Berthoz y Hill [14] estudiaron la validez del uso de autoinformes para valorar la regulación emocional en adultos con trastornos del espectro autista y hallaron que los cuestionarios autoinformativos sobre regulación emocional pueden utilizarse con personas con trastornos del espectro autista para caracterizar su estilo afectivo, aunque dichos datos autobiográficos se expresaban de una manera peculiar. A la luz de los datos de Baron-Cohen et al [15], la fiabilidad de los autoinformes ofrecidos por sujetos con trastornos del espectro autista es adecuada. No obstante, debemos ser cautos con los resultados de 
todos estos estudios debido a la muestra pequeña con la que se han desarrollado.

Los resultados del estudio son muy significativos cuando se trata de valorar los niveles de alexitimia en los sujetos con SA por parte de las personas que conviven con ellos, sus familiares. Son éstos quienes experimentan cotidianamente el impacto que tiene en sus relaciones interpersonales la alexitimia de su familiar con SA: poca calidez en la relación, dificultad para sentir compasión, poca sensibilidad, rigidez en la vida cotidiana, confusión ante el estrés, frustración al enfrentarse a lo incierto, poco sentido del humor, etc.

La prospectiva de la investigación apunta a cómo explicar la alta comorbilidad entre alexitimia y SA. ¿Se trata la alexitimia de una consecuencia estructural neuroanatómica o es fruto de una deficiencia neurobiológica?, ¿es más bien de un rasgo de personalidad idiosincrásico de los sujetos con SA? Si es así, ¿no configuraría, pues, la alexitimia un criterio diagnóstico para el SA? No en vano, los diversos criterios diagnósticos contemplan aspectos relacionados con la alexitimia: 'falta de reciprocidad en áreas sociales y emocionales' (DSM-IV-TR) [5], 'alteración en las respuestas a las emociones de los demás' (ICD-10) [16], 'dificultades para expresarse, especialmente cuando hablan de emociones' (definición de la National Autistic Society) [17], 'comportamientos emocionales inapropiados, [...] alteraciones en su comprensión' (criterios diagnósticos de Gillberg) [18]...

Como dice Attwood [19], 'ahora disponemos de un término psicológico, alexitimia, para describir otra característica asociada al SA; es decir, alguien que tiene una alteración en la habilidad para identificar y describir estados emocionales. La experiencia clínica y la investigación han confirmado que la alexitimia puede reconocerse en el perfil de habilidades de las personas con SA'.
Bibliografía

1. Sifneos PE. The prevalence of 'alexithymic' characteristics in psychosomatic patients. Psychother Psychosom 1973; 22: 255-62.

2. Sifneos PE. Alexithymia and its relationship to hemispheric specialization, affect, and creativity. Psychiatr Clin North Am 1988; 11: 287-92.

3. Thompson J. Emotionally dumb: an overview of alexithymia London: Jessica Kingsley Publishers; 2009.

4. Taylor GJ, Bagby RM, Parker JDA. Disorders of affect regulation. Alexithymia in medical and psychiatric illness. Cambridge: Cambridge University Press; 1997.

5. DSM-IV. Manual diagnóstico y estadístico de los trastornos mentales. Barcelona: Masson; 1995.

6. Bagby RM, Parker JDA, Taylor GJ. The twenty-item Toronto Alexithymia Scale-I. Item selection and cross-validation of the factor structure. J Psychosom Res 1994; 38: 23-32.

7. Moral de la Rubia, J, Retamales, R. Estudio de validación de la escala de alexitimia de Toronto (TAS-20) en muestra española. Psiquiatría.com 2000; 4: 1-10.

8. Martínez-Sánchez, F. Adaptación española de la escala de alexitimia de Toronto (TAS-20). Clínica y Salud 1996; 7: 19-32.

9. Thomson J. Cuestionario de alexitimia en línea (OAQ-G2). URL: http://oaq.blogspot.com.

10. Haviland MG, Sonne JL, Coger PA. Alexithymia and psychopathy: comparison and application of California Q-set prototypes. J Pers Assess 2004; 82: 306-16.

11. Watson D, Clark LA, Tellegen A. Development and validation of brief measures of positive and negative affect: the PANAS scale. J Pers Soc Psychol 1988; 54: 1063-70.

12. Hill E, Berthoz S, Frith U. Brief report: cognitive processing of own emotions in individuals with autistic spectrum disorder and in their relatives. J Autism Dev Disord 2004; 34: 229-35.

13. Lombardo MV, Chakrabarti B, Bullmore ET, Sadek SA, Pasco G, Wheelwright SJ. Atypical neural self-representation in autism. Brain 2009; Dec 13, Epub ahead of print.

14. Berthoz S, Hill EL. The validity of using self-reports to assess emotion regulation abilities in adults with autism spectrum disorder. Eur Psychiatry 2005; 20: 291-8.

15. Baron-Cohen S, Wheelwright S, Skinner R, Martin J, Cluble E. The autism-spectrum quotient (AQ): evidence from Asperger syndrome/high-functioning autism, males and females, mathematicians and scientists. J Autism Dev Disord 2001; 31: 5-17.

16. International Classification of Diseases (ICD-10). Geneva: World Health Organization; 1992.

17. National Autistic Society (NAS). URL: http://www.nas.org.uk

18. Gillberg CI, Gillberg C. Asperger syndrome. Some epidemiological considerations: a research note. J Child Psychol Psychiatry 1989; 30: 631-8.

19. Attwood T. The complete guide to Asperger's syndrome. London: Jessica Kingsley Publishers; 2006.

\section{Alexithymia and Asperger syndrome}

Introduction. The study starts with the hypothesis that the difficulty to identify and describe emotions and feelings, and to differentiate the feelings of bodily sensations that accompany them, are a common denominator of the construct of alexithymia and Asperger syndrome (AS).

Patients and methods. The study evaluates the levels of alexithymia in nine adult male subjects with AS.

Results. The results are classified according to whether the source of information is the subject itself with SA (significant alexithymia level in $2 / 3$ parts of the subjects surveyed) or if the source is a relative (the final score in relation to the presence of the alexithymia is the double than in neurotypical population).

Conclusion. It's confirmed a significant comorbidity between alexithymia and SA leading to the question of whether alexithymia is a idiosyncratic trait of individuals with SA.

Key words. Adults with Asperger syndrome. Alexithymia. 